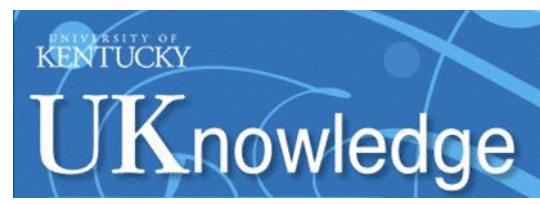

University of Kentucky

UKnowledge

9-1-2017

\title{
On $\tau_{\sigma}$ Quasinormal Subgroups of Finite Groups
}

James C. Beidleman

University of Kentucky, clark@ms.uky.edu

Alexander N. Skiba

Francisk Skorina Gomel State University, Belarus

Follow this and additional works at: https://uknowledge.uky.edu/math_facpub

Part of the Mathematics Commons

Right click to open a feedback form in a new tab to let us know how this document benefits you.

\section{Repository Citation}

Beidleman, James C. and Skiba, Alexander N., "On $\tau_{\sigma}$ Quasinormal Subgroups of Finite Groups" (2017).

Mathematics Faculty Publications. 29.

https://uknowledge.uky.edu/math_facpub/29

This Article is brought to you for free and open access by the Mathematics at UKnowledge. It has been accepted for inclusion in Mathematics Faculty Publications by an authorized administrator of UKnowledge. For more information, please contact UKnowledge@lsv.uky.edu. 
On $\tau_{\sigma}$ Quasinormal Subgroups of Finite Groups

Digital Object Identifier (DOI)

https://doi.org/10.1515/jgth-2017-0016

Notes/Citation Information

Published in Journal of Group Theory, v. 20, issue 5, p. 955-969.

๑ de Gruyter 2017

The copyright holder has granted the permission for posting the article here.

This article is available at UKnowledge: https://uknowledge.uky.edu/math_facpub/29 


\title{
On $\tau_{\sigma}$-quasinormal subgroups of finite groups
}

\author{
James C. Beidleman and Alexander N. Skiba \\ Communicated by Evgenii I. Khukhro
}

\begin{abstract}
Let $\sigma=\left\{\sigma_{i} \mid i \in I\right\}$ be a partition of the set of all primes $\mathbb{P}$ and $G$ a finite group. A set $\mathscr{H}$ of subgroups of $G$ is said to be a complete Hall $\sigma$-set of $G$ if every member $\neq 1$ of $\mathscr{H}$ is a Hall $\sigma_{i}$-subgroup of $G$ for some $i \in I$ and $\mathscr{H}$ contains exactly one Hall $\sigma_{i}$-subgroup of $G$ for every $i$ such that $\sigma_{i} \cap \pi(G) \neq \varnothing$.

Let $\tau_{\mathscr{H}}(A)=\left\{\sigma_{i} \in \sigma(G) \backslash \sigma(A) \mid \sigma(A) \cap \sigma\left(H^{G}\right) \neq \emptyset\right.$ for a Hall $\sigma_{i}$-subgroup $H$ of $\left.G\right\}$. We say that a subgroup $A$ of $G$ is $\tau_{\sigma}$-permutable or $\tau_{\sigma}$-quasinormal in $G$ with respect to $\mathscr{H}$ if $A H^{x}=H^{x} A$ for all $x \in G$ and all $H \in \mathscr{H}$ such that $\sigma(H) \subseteq \tau_{\mathscr{H}}(A)$, and $\tau_{\sigma}$-permutable or $\tau_{\sigma}$-quasinormal in $G$ if $A$ is $\tau_{\sigma}$-permutable in $G$ with respect to some complete Hall $\sigma$-set of $G$.
\end{abstract}

We study $G$ assuming that $\tau_{\sigma}$-quasinormality is a transitive relation in $G$.

\section{Introduction}

Throughout this paper, all groups are finite and $G$ always denotes a finite group. Moreover, $\mathbb{P}$ is the set of all primes, $\pi \subseteq \mathbb{P}$ and $\pi^{\prime}=\mathbb{P} \backslash \pi$. The group $G$ is called $\pi$-supersoluble provided every chief factor of $G$ is either cyclic or a $\pi^{\prime}$-group.

If $n$ is an integer, the symbol $\pi(n)$ denotes the set of all primes dividing $n$; as usual, $\pi(G)=\pi(|G|)$, the set of all primes dividing the order of $G$. The symbol $H_{G}$ denotes the largest normal subgroup of $G$ contained in $H \leq G$.

In what follows, $\sigma$ is some partition of $\mathbb{P}$, that is, $\sigma=\left\{\sigma_{i} \mid i \in I\right\}$, where $\mathbb{P}=\bigcup_{i \in I} \sigma_{i}$ and $\sigma_{i} \cap \sigma_{j}=\emptyset$ for all $i \neq j$.

The symbol $\sigma(n)$ denotes the set $\left\{\sigma_{i} \mid \sigma_{i} \cap \pi(n) \neq \emptyset\right\} ; \sigma(G)=\sigma(|G|)$. The group $G$ is said to be

(i) $\sigma$-primary [16] if $G$ is a $\sigma_{i}$-group for some $i \in I$,

(ii) $\sigma$-decomposable (Shemetkov [14]) or $\sigma$-nilpotent (Guo and Skiba [6]) if $G=G_{1} \times \cdots \times G_{n}$ for some $\sigma$-primary groups $G_{1}, \ldots, G_{n}$,

(iii) $\sigma$-soluble [16] if every chief factor of $G$ is $\sigma$-primary.

A set $\mathscr{H}$ of subgroups of $G$ is a complete Hall $\sigma$-set of $G[15,17]$ if every member $\neq 1$ of $\mathscr{H}$ is a Hall $\sigma_{i}$-subgroup of $G$ for some $\sigma_{i} \in \sigma$ and $\mathscr{H}$ contains exactly one Hall $\sigma_{i}$-subgroup of $G$ for every $\sigma_{i} \in \sigma(G)$. 
Let $\tau_{\mathscr{H}}(A)=\left\{\sigma_{i} \in \sigma(G) \backslash \sigma(A) \mid \sigma(A) \cap \sigma\left(H^{G}\right) \neq \emptyset\right.$ for a Hall $\sigma_{i}$-subgroup $H \in \mathscr{H}\}$.

Definition 1.1. We say that a subgroup $A$ of $G$ is

(i) $\tau_{\sigma}$-permutable or $\tau_{\sigma}$-quasinormal in $G$ with respect to $\mathscr{H}$ if $A H^{x}=H^{x} A$ for all $x \in G$ and all $H \in \mathscr{H}$ such that $\sigma(H) \subseteq \tau_{\mathscr{H}}(A)$,

(ii) $\tau_{\sigma}$-permutable or $\tau_{\sigma}$-quasinormal in $G$ if $A$ is $\tau_{\sigma}$-permutable in $G$ with respect to some complete Hall $\sigma$-set $\mathscr{H}$ of $G$.

Recall that a subgroup $A$ of $G$ is said to be

(i) $\sigma$-permutable or $\sigma$-quasinormal in $G$ if $G$ possesses a complete Hall $\sigma$-set $\mathscr{H}$ such that $A H^{x}=H^{x} A$ for all $H \in \mathscr{H}$ and all $x \in G$ (cf. [16]),

(ii) $\sigma$-semipermutable in $G$ if $G$ possesses a complete Hall $\sigma$-set $\mathscr{H}$ such that $A H^{x}=H^{x} A$ for all $x \in G$ and all $H \in \mathscr{H}$ with $\sigma(A) \cap \sigma(H)=\emptyset$ (cf. [7]).

In the classical case when $\sigma=\{\{2\},\{3\}, \ldots\}, \sigma$-permutable, $\sigma$-semipermutable and $\tau_{\sigma}$-quasinormal subgroups are also called respectively $S$-permutable $[1,5]$, $S$-semipermutable [5] and $\tau$-quasinormal $[11,12]$.

It is clear that every $\sigma$-quasinormal subgroup is also $\sigma$-semipermutable and every $\sigma$-semipermutable subgroup is $\tau_{\sigma}$-quasinormal.

Example 1.2. Let $p>q>r$ be primes, $C_{r}$ a group of order $r$ and $H=Q \rtimes C_{r}$, where $Q$ is a simple $\mathbb{F}_{q} C_{r}$-module which is faithful for $C_{r}$. Let $G=P \rtimes H$, where $P$ is a simple $\mathbb{F}_{p} H$-module which is faithful for $H$. Let $\sigma=\left\{\sigma_{1}, \sigma_{2}, \sigma_{3}\right\}$, where $\sigma_{1}=\{p\}, \sigma_{2}=\{q\}$, and $\sigma_{3}=\{p, q\}^{\prime}$. Then every Sylow $r$-subgroup of $G$ is $\tau_{\sigma}$-quasinormal but not $\sigma$-semipermutable. A Hall $\{q, r\}$-subgroup of $G$ is $\sigma$-semipermutable in $G$ but not $\sigma$-quasinormal.

We say that $G$ is a $T \sigma T$-group if $\tau_{\sigma}$-quasinormality is a transitive relation in $G$, that is, if $K$ is a $\tau_{\sigma}$-quasinormal subgroup of $H$ and $H$ is a $\tau_{\sigma}$-quasinormal subgroup of $G$, then $K$ is a $\tau_{\sigma}$-quasinormal subgroup of $G$. Our purpose here is to establish the structure of $\sigma$-soluble $T \sigma T$-groups.

Theorem A. Let $D=G^{\mathfrak{N}_{\sigma}}$ and $\pi=\pi(D)$. Suppose that $G$ possesses a complete Hall $\sigma$-set $\mathscr{H}$ such that all members of $\mathscr{H}$ are $\pi$-supersoluble. Then $G$ is a $\sigma$-soluble $T \sigma T$-group if and only if either $G$ is $\sigma$-nilpotent or $G=D \rtimes M$, where:

(i) $D$ and $M$ are Hall subgroups of $G$ and the smallest prime divisor of $|G|$ divides $|M|$.

(ii) $D$ is abelian and every element of $M$ induces a power automorphism in $D$. 
(iii) Every subgroup of $G$ is $\tau_{\sigma}$-quasinormal in $G$.

(iv) If $A$ and $B$ are respectively a Hall $\sigma_{i}$-subgroup and a Hall $\sigma_{j}$-subgroup of $G$, where $i \neq j$ and $\sigma_{i}, \sigma_{j} \in \sigma(G) \backslash \sigma(D)$, then the order of $[A, B]$ divides a prime. Moreover, if $|[A, B]|=r \neq 1$, then $r \in \pi(D)$ and either the Sylow $r$-subgroup $R$ of $G$ is cyclic or $[A, R]=1=[B, R]$; if, also, $A$ is a $p$-group and $B$ is a q-group for some primes $p$ and $q$, then $r>p$ and $r>q$.

In this theorem $G^{\mathfrak{N}_{\sigma}}$ denotes the $\sigma$-nilpotent residual of $G$, that is, the intersection of all normal subgroups $N$ of $G$ with $\sigma$-nilpotent quotient $G / N$.

One of the main objectives of this paper is to give a correct proof of [12, Theorem 1.1]. The proof given in [12] for this theorem has several gaps. However, Theorem A and its proof allows us to eliminate all of those gaps. Corollary 1.3, our next result, is a statement of [12, Theorem 1.1].

Recall that $G$ is said to be a TQT-group [12] if $\tau$-quasinormality is a transitive relation in $G$.

Corollary 1.3. Let $P$ be a Sylow p-subgroup of $G, Q$ a Sylow $q$-subgroup of $G$ such that $p \neq q$. The following statements are equivalent:

(1) $G$ is a soluble $T Q T$-group.

(2) $G$ is a supersoluble group which has an abelian normal Hall subgroup of odd order $D$ such that $G / D$ is nilpotent, every subgroup of $D$ is normal in $G$, and every subgroup of $G$ is $\tau$-quasinormal in $G$. Moreover, if $p \notin \pi(D)$ and $q \notin \pi(D)$, then the order of $[P, Q]$ divides $r$, where $r \in \pi(D)$, and if $|[P, Q]|=r$, then $r>p, r>q$ and either the Sylow $r$-subgroup $R$ of $G$ is cyclic or $[P, R]=1=[Q, R]$.

The following observation covers many steps in the proof of Theorem A.

Theorem B. Let $D=G^{\mathfrak{N}_{\sigma}}$ and $\pi=\pi(D)$. Suppose that $G$ possesses a complete Hall $\sigma$-set $\mathscr{H}$ such that all members of $\mathscr{H}$ are $\pi$-supersoluble. If all maximal subgroups of every Sylow p-subgroup of $G$ are $\tau_{\sigma}$-quasinormal in $G$ for all $p \in \pi$, then $D$ is a nilpotent Hall subgroup of $G$, the smallest prime divisor of $|G|$ divides $|G: D|$ and every chief factor of $G$ below $D$ is cyclic.

Corollary 1.4 (Srinivasan [18]). If every maximal subgroup of every Sylow subgroup of $G$ is $S$-permutable in $G$, then $G$ is supersoluble.

\section{Preliminaries}

We use $\mathfrak{N}_{\sigma}$ to denote the class of all $\sigma$-nilpotent groups. 
Lemma 2.1 (see [16, Lemma 2.5]). The class $\mathfrak{N}_{\sigma}$ is closed under taking direct products, homomorphic images and subgroups. Moreover, if $E$ is a normal subgroup of $G$ and $E / E \cap \Phi(G)$ is $\sigma$-nilpotent, then $E$ is $\sigma$-nilpotent.

In view of [2, Proposition 2.2.8], we get from Lemma 2.1 the following:

Lemma 2.2. If $N$ is a normal subgroup of $G$, then

$$
(G / N)^{\mathfrak{\Re}_{\sigma}}=G^{\mathfrak{\Re}_{\sigma}} N / N .
$$

Lemma 2.3 (Knyagina and Monakhov [10]). Let $H, K$ and $N$ be pairwise permutable subgroups of $G$ and $H$ is a Hall subgroup of $G$. Then

$$
N \cap H K=(N \cap H)(N \cap K) .
$$

Suppose that $G$ has a complete Hall $\sigma$-set $\mathscr{H}=\left\{H_{1}, \ldots, H_{t}\right\}$. For any subgroup $H$ of $G$ we write $H \cap \mathscr{H}$ to denote the set $\left\{H \cap H_{1}, \ldots, H \cap H_{t}\right\}$. If $H \cap \mathscr{H}$ is a complete Hall $\sigma$-set of $H$, then we say that $\mathscr{H}$ reduces into $H$.

Recall that $G$ is said to be

(i) a $D_{\pi}$-group if $G$ possesses a Hall $\pi$-subgroup $E$ and every $\pi$-subgroup of $G$ is contained in some conjugate of $E$,

(ii) a $\sigma$-full group of Sylow type [16] if every subgroup $E$ of $G$ is a $D_{\sigma_{i}}$-group for every $\sigma_{i} \in \sigma(E)$,

(iii) $\sigma$-full $[15,17]$ provided $G$ possesses a complete Hall $\sigma$-set.

In view of $[15$, Theorems $A$ and $B]$, the following fact is true.

Lemma 2.4. If $G$ is $\sigma$-soluble, then $G$ is a $\sigma$-full group of Sylow type.

Lemma 2.5 (see [16, Lemma 3.1]). Let $H$ be a $\sigma_{i}$-subgroup of a $\sigma$-full group $G$. Then $H$ is $\sigma$-permutable in $G$ if and only if $O^{\sigma_{i}}(G) \leq N_{G}(H)$.

Lemma 2.6. Suppose that $G$ has a complete Hall $\sigma$-set $\mathscr{H}=\left\{H_{1}, \ldots, H_{t}\right\}$ such that the subgroups $H$ and $K$ of $G$ are $\tau_{\sigma}$-quasinormal in $G$ with respect to $H$. Let $R$ be a normal subgroup of $G$ and $H \leq L \leq G$. Then:

(1) $\mathscr{H}_{0}=\left\{H_{1} R / R, \ldots, H_{t} R / R\right\}$ is a complete Hall $\sigma$-set of $G / R$. Moreover, if $\sigma(H)=\sigma(H R / R)$, then $H R / R$ is $\tau_{\sigma}$-quasinormal in $G / N$ with respect to $\mathscr{H}_{0}$.

(2) If $H K=K H$ and $\sigma(H \cap K)=\sigma(H)=\sigma(K)$, then $H \cap K$ is $\tau_{\sigma}$-quasinormal in $G$ with respect to $\mathcal{H}$.

(3) If for some $i$ we have $H \leq O_{\sigma_{i}}(G)$, then $H$ is $\sigma$-quasinormal in $G$. 
(4) If $\mathscr{H}$ reduces into $L$, then $H$ is $\tau_{\sigma}$-quasinormal in $L$ with respect to $L \cap \mathscr{H}$.

(5) If $G$ is a $\sigma$-full group of Sylow type, then $H$ is $\tau_{\sigma}$-quasinormal in $L$.

Proof. Without loss of generality we can assume that $H_{i}$ is a $\sigma_{i}$-group for all $i=1, \ldots, t$.

(1) It is clear that $\mathscr{H}_{0}=\left\{H_{1} R / R, \ldots, H_{t} R / R\right\}$ is a complete Hall $\sigma$-set of $G / R$. Now let $\sigma_{i} \in \tau_{\mathcal{H}_{0}}(H R / R)$, that is, $\sigma_{i} \notin \sigma(H R / R)$ and

$$
\sigma(H R / R) \cap \sigma\left(\left(H_{i} R / R\right)^{G}\right) \neq \emptyset .
$$

Then $\sigma_{i} \notin \sigma(H)$ since $\sigma(H)=\sigma(H R / R)$. On the other hand, we have

$$
\left(H_{i} R / R\right)^{G}=H_{i}^{G} R / R \simeq H_{i}^{G} /\left(H_{i}^{G} \cap R\right),
$$

so $\sigma(H) \cap \sigma\left(H_{i}^{G}\right) \neq \emptyset$. Hence $\sigma_{i} \in \tau_{\mathscr{H}}(H)$ and so

$$
\begin{aligned}
(H R / R)\left(H_{i} R / R\right) & =H H_{i} R / R \\
& =H_{i} H R / R \\
& =\left(H_{i} R / R\right)(H R / R) .
\end{aligned}
$$

Thus $H R / R$ is $\tau_{\sigma}$-quasinormal in $G / R$ with respect to $\mathscr{H}_{0}$.

(2) Let $H_{i}$ be a member of $\mathscr{H}$ such that $\sigma_{i} \in \tau_{\mathscr{H}}(H \cap K)$, that is, we have $\sigma_{i} \in \sigma(G) \backslash \sigma(H \cap K)$ and $\sigma(H \cap K) \cap \sigma\left(H_{i}^{G}\right) \neq \emptyset$. Then $\sigma_{i} \in \sigma(G) \backslash \sigma(H)$ (since $\sigma(H \cap K)=\sigma(H)$ ) and $\sigma(H) \cap \sigma\left(H_{i}^{G}\right) \neq \emptyset$, so $\sigma_{i} \in \tau_{\mathscr{H}}(H)$. Similarly we get that $\sigma_{i} \in \tau_{\mathscr{H}}(K)$. Hence $H H_{i}^{x}=H_{i}^{x} H$ and $K H_{i}^{x}=H_{i}^{x} K$ for all $x \in G$. It is clear also that $H \cap H_{i}^{x}=1$. Therefore for every $x \in G$ we have

$$
\begin{aligned}
H H_{i}^{x} \cap K H_{i}^{x} & =H_{i}^{x}\left(H \cap K H_{i}^{x}\right) \\
& =H_{i}^{x}(H \cap K)\left(H \cap H_{i}^{x}\right) \\
& =H_{i}^{x}(H \cap K) \\
& =(H \cap K) H_{i}^{x}
\end{aligned}
$$

by Lemma 2.3. Hence $H \cap K$ is $\tau_{\sigma}$-quasinormal in $G$ with respect to $\mathscr{H}$.

(3) Let $j \neq i$. Suppose that for some $x \in G$ we have $H H_{j}^{x} \neq H_{j}^{x} H$. Then $\sigma_{j} \notin \tau_{\mathscr{H}}(H)$. Hence $\sigma(H) \cap \sigma\left(H_{j}^{G}\right)=\emptyset$ since $H$ is $\tau_{\sigma}$-quasinormal in $G$ with respect to $\mathscr{H}$ by hypothesis. But then $H_{j}^{G}$ is a $\sigma_{i}^{\prime}$-group, so $H \leq O_{\sigma_{i}}(G) \leq$ $C_{G}\left(H_{j}^{G}\right)$, which implies that $H H_{j}^{x}=H_{j}^{x} H$. This contradiction shows that we have (3).

(4) Let $L_{i}=H_{i} \cap L$ for all $i=1, \ldots, t$ and $\mathscr{L}=\left\{L_{1}, \ldots, L_{t}\right\}$. By hypothesis, $\mathscr{L}$ is a complete Hall $\sigma$-set of $L$. Let $\sigma_{i} \in \tau_{\mathscr{L}}(H)$, that is, $\sigma_{i} \in \sigma(L) \backslash \sigma(H)$ and $\sigma(H) \cap \sigma\left(\left(L_{i}\right)^{L}\right) \neq \emptyset$. Then $\sigma_{i} \in \sigma(G) \backslash \sigma(H)$ and $\sigma(H) \cap \sigma\left(\left(H_{i}\right)^{G}\right) \neq \varnothing$ 
since $\left(L_{i}\right)^{L} \leq L_{i}^{G} \leq\left(H_{i} \cap L\right)^{G} \leq H_{i}^{G}$. Hence $\sigma_{i} \in \tau_{\mathscr{H}}(H)$, so $H H_{i}^{a}=H_{i}^{a} H$ for all $a \in L$, so $L \cap H H_{i}^{a}=H\left(L \cap H_{i}^{a}\right)=H\left(L \cap H_{i}\right)^{a}=H L_{i}^{a}=L_{i}^{a} H$. This shows that $H$ is $\tau_{\sigma}$-quasinormal in $L$ with respect to $L \cap \mathscr{H}$.

(5) Since $G$ is a $\sigma$-full group of Sylow type, $H$ is $\tau_{\sigma}$-quasinormal in $G$ with respect to each complete Hall $\sigma$-set of $G$. Moreover, this condition implies also that some complete Hall $\sigma$-set of $G$ reduces into $L$, so we (5) by part (4).

The lemma is proved.

Lemma 2.7 (Kegel [9]). Let $A$ and $B$ be subgroups of $G$ such that $G \neq A B$ and $A B^{x}=B^{x} A$ for all $x \in G$. Then $G$ has a proper normal subgroup $N$ such that either $A \leq N$ or $B \leq N$.

The following lemma is a corollary of [4, Chapter IV, (6.7)] (see also [3, Lemma 2.12]).

Lemma 2.8. Let $N \leq E$ be normal subgroups of $G$ such that $N \leq \Phi(E)$ and every chief factor of $G$ between $E$ and $N$ is cyclic. Then every chief factor of $G$ below $E$ is cyclic.

Lemma 2.9. If $\mathscr{H}=\left\{H_{1}, \ldots, H_{t}\right\}$ is a complete Hall $\sigma$-set of $G$ and every member of $\mathscr{H}$ is $\pi\left(G^{\mathfrak{N}_{\sigma}}\right)$-supersoluble, then

$$
\mathscr{H}_{0}=\left\{H_{1} N / N, \ldots, H_{t} N / N\right\}
$$

is a complete Hall $\sigma$-set of $G / N$ and every member of $\mathcal{H}_{0}$ is $\pi\left((G / N)^{\mathfrak{N}_{\sigma}}\right)$-supersoluble.

Proof. It is clear that $\mathscr{H}_{0}$ is a complete Hall $\sigma$-set of $G / N$. Now let $D=G^{\mathfrak{R}_{\sigma}}$ and $\pi=\pi(D)$. Then $(G / N)^{\mathfrak{R}_{\sigma}}=D N / N$ by Lemma 2.2 , so

$$
\pi_{0}=\pi\left((G / N)^{\mathfrak{\Re}_{\sigma}}\right)=\pi(D N / N)=\pi(D / D \cap N) \subseteq \pi(D)=\pi .
$$

Hence every member $H_{i}$ of $\mathscr{H}$ is $\pi_{0}$-supersoluble, so $H_{i} N / N$ is $\pi_{0}$-supersoluble. The lemma is proved.

Lemma 2.10. Let $D=G^{\mathfrak{N}_{\sigma}}$ and $\pi=\pi(D)$. Suppose that $D$ is a nilpotent Hall subgroup of $G$ and $G$ possesses a complete Hall $\sigma$-set $\mathscr{H}$ such that all members of $\mathscr{H}$ are $\pi$-supersoluble. If every $\sigma_{i}$-subgroup of $G$ is $\tau_{\sigma}$-quasinormal in $G$ for all $\sigma_{i} \in \sigma(D)$, then every subgroup of $D$ is normal in $G$.

Proof. Suppose that this lemma is false and let $G$ be a counterexample of minimal order. Let $\mathscr{H}=\left\{H_{1}, \ldots, H_{t}\right\}$. We can assume without loss of generality that $H_{i}$ is a $\sigma_{i}$-group for all $i=1, \ldots, t$. 
We show that the hypothesis holds on $G / N$ for every minimal normal subgroup $N$ of $G$. First note that

$$
(G / N)^{\mathfrak{\Re}_{\sigma}}=D N / N \simeq D / D \cap N
$$

is a nilpotent Hall subgroup of $G / N$ by Lemma 2.2, and $G / N$ possesses a complete Hall $\sigma$-set $\mathscr{H}_{0}$ such that all members of $\mathscr{H}_{0}$ are $\pi\left((G / N)^{\mathfrak{N}_{\sigma}}\right)$-supersoluble by Lemma 2.9 .

Now let $V / N$ be a non-identity $\sigma_{i}$-subgroup of $G / N$ for some

$$
\sigma_{i} \in \sigma\left((G / N)^{\mathfrak{\Re}_{\sigma}}\right)=\sigma(D N / N)=\sigma(D / D \cap N) \subseteq \sigma(D) .
$$

And let $U$ be a minimal supplement to $N$ in $V$. Then $U \cap N \leq \Phi(U)$, so $U$ is a $\sigma_{i}$-subgroup of $G$ since $V / N=U N / N \simeq U / U \cap N$. Therefore $U$ is $\tau_{\sigma}$-quasinormal in $G$ by hypothesis and $\sigma(U)=\sigma(U N / N)=\left\{\sigma_{i}\right\}$, which implies that $V / N=U N / N$ is $\tau_{\sigma}$-quasinormal in $G / N$ by Lemma $2.6(1)$. Hence the hypothesis holds on $G / N$.

Let $H$ be a subgroup of the Sylow $p$-subgroup $P$ of $D$ for some prime $p \in \pi$. We show that $H$ is normal in $G$. For some $i$ we have $P \leq O_{\sigma_{i}}(D)=H_{i} \cap D$. On the other hand, we have $D=O_{\sigma_{i}}(D) \times O^{\sigma_{i}}(D)$ since $D$ is nilpotent. Assume that $O^{\sigma_{i}}(D) \neq 1$ and let $N$ be a minimal normal subgroup of $G$ contained in $O^{\sigma_{i}}(D)$. Then $H N / N \leq D N / N=(G / N)^{\mathfrak{\Re}_{\sigma}}$, so the choice of $G$ implies that $H N / N$ is normal in $G / N$. Hence $H=H\left(N \cap O_{\sigma_{i}}(D)\right)=H N \cap O_{\sigma_{i}}(D)$ is normal in $G$.

Now assume that $O^{\sigma_{i}}(D)=1$, so $D$ is a $\sigma_{i}$-group. Since $G / D$ is $\sigma$-nilpotent by Lemma 2.1, $H_{i} / D$ is normal in $G / D$ and hence $H_{i}$ is normal in $G$. Therefore all subgroups of $H_{i}$ are $\sigma$-quasinormal in $G$ by Lemma 2.6(3) and hypothesis. Since $D$ is a normal Hall subgroup of $H_{i}$, it has a complement $S$ in $H_{i}$ by the Schur-Zassenhaus theorem. Lemma 2.5 implies that $D \leq O^{\sigma_{i}}(G) \leq N_{G}(S)$. Hence $H_{i}=D \times S$. Therefore

$$
G=H_{i} O^{\sigma_{i}}(G)=S O^{\sigma_{i}}(G) \leq N_{G}(H),
$$

so $H$ is normal in $G$.

Therefore every subgroup of $D$ is normal in $G$ since $D$ is nilpotent by hypothesis. The lemma is proved.

\section{Proof of Theorems A and B}

Proof of Theorem B. Suppose that this theorem is false and let $G$ be a counterexample of minimal order. Then $D \neq 1$. Let $\mathscr{H}=\left\{H_{1}, \ldots, H_{t}\right\}$. We can assume without loss of generality that $H_{i}$ is a $\sigma_{i}$-group for all $i=1, \ldots, t$. Let $R$ be a minimal normal subgroup of $G$. 
Claim 1. The conclusion of the theorem holds for $G / R$.

Let $V / R$ be a maximal subgroup of a Sylow $p$-subgroup $P / R$ of $G / R$, where $p \in \pi\left((G / R)^{\mathfrak{R}_{\sigma}}\right)$. Then for some Sylow $p$-subgroup $G_{p}$ of $G$ we have $P / R=$ $G_{p} R / R$ and $V=R\left(V \cap G_{p}\right)$. It is clear that $V \cap G_{p}$ is a maximal subgroup of $G_{p}$. Therefore $V \cap G_{p}$ is $\tau_{\sigma}$-quasinormal in $G$ by hypothesis since

$$
\pi\left((G / R)^{\Re_{\sigma}}\right)=\pi(D R / R)=\pi(D / D \cap R) \subseteq \pi
$$

by Lemma 2.2 , so $V / R=R\left(V \cap G_{p}\right) / R$ is $\tau_{\sigma}$-quasinormal in $G / R$ by Lemma 2.6(1). Consequently, the hypothesis holds for $G / R$ by Lemma 2.9. Hence we have Claim 1 by the choice of $G$.

Claim 2. The group $D$ is soluble, so $G$ is $\sigma$-soluble. Hence $G$ is a $\sigma$-full group of Sylow type.

Assume that this is false. By Claim 1 and Lemma 2.2,

$$
(G / R)^{\mathfrak{N}_{\sigma}}=D R / R \simeq D / D \cap R
$$

is nilpotent. Hence $R \leq D$. Moreover, if $G$ has a minimal normal subgroup $N \neq R$, then $N \leq D$ and $D \simeq D / R \cap N=D / 1$ is nilpotent. Therefore $C_{G}(R)=1$. Then 2 divides $|R|$ by the Feit-Thompson theorem, and a Sylow 2-subgroup $Q$ of $R$ is not cyclic by [8, Chapter IV, Section 2.8]. Hence $|Q|>2$.

Let $P$ be a Sylow 2-subgroup of $G$ such that $Q=P \cap R$. Then for some maximal subgroup $V$ of $P$ we have $Q \not \leq V$ by the Tate theorem [8, Chapter IV, Section 4.7], which implies that $P=Q V$ and so $V \cap R<P \cap R=Q$. Moreover, $V \cap R \neq 1$ since otherwise we have $V \cap R=P \cap V \cap R=Q \cap V=1$ and so $|Q|=2$. Since $R=R_{1} \times \cdots \times R_{n}$, where $R_{1} \simeq \cdots \simeq R_{n}$ are non-abelian simple groups, it follows that $Q=\left(P \cap R_{1}\right) \times \cdots \times\left(P \cap R_{n}\right)$ and so for some $i$ we have $V \cap R_{i}<P \cap R_{i}$. Note also that $V \cap R_{i} \neq 1$ since otherwise from the isomorphism

$$
V\left(P \cap R_{i}\right) / V \simeq\left(P \cap R_{i}\right) /\left(V \cap\left(P \cap R_{i}\right)\right)=P \cap R_{i} / 1
$$

we get that the order of a Sylow 2-subgroup of $P \cap R_{i}$ divides 2 and so $P \cap R_{i}$ is 2-nilpotent by [8, Chapter IV, Section 2.8], which implies that $R$ is 2-nilpotent.

Assume that $2 \in \sigma_{k}$. First we show that $R$ is $\sigma$-primary. Suppose that this is false. We can assume without loss of generality that $V$ is $\tau_{\sigma}$-quasinormal in $G$ with respect to $\mathcal{H}$. Then for some $j \neq k$ and for $H=H_{j}$ we have $H \cap R_{i} \neq 1$ since $R$ is not $\sigma$-primary. Note also that $\sigma_{k} \in \sigma\left(H^{G}\right)$ since otherwise we have $R \cap H^{G}=1$, which implies that $1<H^{G} \leq C_{G}(R)=1$. Therefore $\sigma_{k} \in \tau_{\mathcal{H}}(V)$, so $V H^{x}=H^{x} V$ for all $x \in G$. By [4, Chapter A, Section 14.1(a)], we have $L=V H^{x} \cap R_{i}$ is a subnormal subgroup of $V H^{x}$, where $V$ is a Hall $\sigma_{k}$-subgroup of $V H^{x}$ and $H^{x}$ is a Hall $\sigma_{j}$-subgroup of $V H^{x}$. Therefore, $L=(L \cap V)\left(L \cap H^{x}\right)$ 
by [4, Chapter I, Section 3.2]. Hence

$$
\begin{aligned}
L & =(L \cap V)\left(L \cap H^{x}\right) \\
& =\left(V H^{x} \cap R_{i} \cap V\right)\left(V H^{x} \cap R_{i} \cap H^{x}\right) \\
& =\left(R_{i} \cap V\right)\left(R_{i} \cap H^{x}\right) \\
& =\left(V \cap R_{i}\right)\left(H \cap R_{i}\right)^{x} \\
& =\left(H \cap R_{i}\right)^{x}\left(V \cap R_{i}\right)
\end{aligned}
$$

for all $x \in R_{i}$, where $\left(H \cap R_{i}\right)\left(V \cap R_{i}\right) \neq R_{i}$ since $V \cap R_{i}<P \cap R_{i}$. Therefore, $R_{i}$ is not simple by Lemma 2.7 since $H \cap R_{i} \neq 1$ and $V \cap R_{i} \neq 1$. This contradiction shows that $R$ is $\sigma$-primary.

Now assume that $R \leq D$ and $R$ is not abelian. Then

$$
\sigma(V \cap R)=\sigma(V)=\sigma(R)=\left\{\sigma_{k}\right\}
$$

since $R$ is $\sigma$-primary, $2 \in \sigma_{k}$ and $V \cap R \neq 1$. Therefore $V \cap R$ is $\tau_{\sigma}$-quasinormal in $G$ by Lemma 2.6(2). But $V \cap R \leq R \leq O_{\sigma_{k}}(G)$ and so $V \cap R$ is $\sigma$-quasinormal in $G$ by Lemma 2.6(3). Hence $R \leq N_{G}(V \cap R)$ by Lemma 2.5 since $R \leq D \leq O^{\sigma_{i}}(G)$. Therefore $V \cap R \leq O_{2}(R)=1$, a contradiction. Thus $R$ is abelian. Hence $D$ is soluble by Claim 1 . Therefore $G$ is $\sigma$-soluble and so $G$ is a $\sigma$-full group of Sylow type by Lemma 2.4.

Claim 3. The group D is nilpotent.

Assume that this is false. Note that $R D / R=(G / R)^{\mathfrak{N}}$ is nilpotent by Claim 1 . Therefore $R \leq D, R$ is the unique minimal normal subgroup of $G$ and $R \not \leq \Phi(G)$ by Lemma 2.1. Claim 2 implies that $R$ is a $p$-group for some prime $p$. Therefore $R=C_{G}(R)$ by [4, Chapter A, Section 15.2], and $G=R \rtimes M$ for some maximal subgroup $M$ of $G$. If $|R|=p$, then $G / C_{G}(R)=G / R$ is a cyclic group. Hence $G$ is supersoluble and therefore $D$ is nilpotent, which contradicts our assumption on $G$. Therefore $|R|>p$.

For some $i$ we have $R \leq H_{i} \cap D$. Then $H_{i}=R \rtimes\left(H_{i} \cap M\right)$ and $H_{i}$ is $\pi$-supersoluble by hypothesis. It follows that some maximal subgroup $V$ of $R$ is normal in $H_{i}$. Let $P$ be a Sylow $p$-subgroup of $H_{i} \cap M$. Then $R P \in \operatorname{Syl}_{p}(G)$ and $V P$ is a maximal subgroup of $R P$, so $V P$ is $\tau_{\sigma}$-quasinormal in $G$ by hypothesis. Then $V=V(R \cap P)=R \cap V P$ is $\sigma$-quasinormal in $G$ by Lemma 2.6(2)-(3). Therefore $O^{\sigma_{i}}(G) \leq N_{G}(V)$ by Lemma 2.5. Hence $G=H_{i} O^{\sigma_{i}}(G) \leq N_{G}(V)$. The minimality of $R$ implies that $V=1$, so $|R|=p$, a contradiction. Hence we have Claim 3.

Claim 4. If $E$ is a subgroup of $G$, then $E^{\mathfrak{N}} \leq D$. 
Note that since $E / E \cap D \simeq E D / D \in \Re$ and $\Re$ is a hereditary class by Lemma 2.1, $E / E \cap D \in \mathfrak{N}$. Hence $E^{\mathfrak{N}} \leq E \cap D$.

Claim 5. The group $D$ is a Hall subgroup of $G$.

Suppose that this is false and let $P$ be a Sylow $p$-subgroup of $D$ such that $1<P<G_{p} \in \operatorname{Syl}_{p}(G)$. We can assume without loss of generality that $G_{p} \leq H_{1}$ and that $R \leq D$.

Claim (a). The group $D=P$ is a minimal normal subgroup of $G$.

Since $D$ is nilpotent by Claim 3, it follows that $R$ is a $q$-group for some prime $q$. Moreover, $D / R=(G / R)^{\mathfrak{R}_{\sigma}}$ is a Hall subgroup of $G / R$ by Claim 1 . Suppose that $P R / R \neq 1$. Then $P R / R \in \operatorname{Syl}_{p}(G / R)$. If $q \neq p$, then $P \in \operatorname{Syl}_{p}(G)$. This contradicts the fact that $P<G_{p}$. Hence we have $q=p$ and so $R \leq P$, therefore $P / R \in \operatorname{Syl}_{p}(G / R)$ and we again get that $P \in \operatorname{Syl}_{p}(G)$. This contradiction shows that $P R / R=1$, which implies that $R=P$ is the unique minimal normal subgroup of $G$ contained in $D$. Since $D$ is nilpotent by Claim 3, a $p^{\prime}$-complement $E$ of $D$ is characteristic in $D$ and so it is normal in $G$. Hence $E=1$, which implies that $R=D=P$.

Claim (b). We have $D \not \leq \Phi(G)$. Hence for some maximal subgroup $M$ of $G$ we have $G=D \rtimes M$.

This follows from Claim 2 and Lemma 2.1 since $G$ is not $\sigma$-nilpotent

Claim (c). If $G$ has a minimal normal subgroup $L \neq D$, then $G_{p}=D \times\left(L \cap G_{p}\right)$. Hence $O_{p^{\prime}}(G)=1$.

Indeed, $D L / L \simeq D$ is a Hall subgroup of $G / L$ by Claims 2 and (a). Hence $G_{p} L / L=D L / L$, so $G_{p}=D \times\left(L \cap G_{p}\right)$. Thus $O_{p^{\prime}}(G)=1$ since $D<G_{p}$ by Claim (a).

Claim (d). The group $V=C_{G}(D) \cap M$ is a normal subgroup of $G$ and we have $C_{G}(D)=D \times V \leq H_{1}$.

In view of Claims (a) and (b), $C_{G}(D)=D \times V$, where $V=C_{G}(D) \cap M$ is a normal subgroup of $G$. By Claim (a), $V \cap D=1$ and hence $V \simeq D V / D$ is $\sigma$-nilpotent by Lemma 2.1. Let $W$ be a $\sigma_{1}$-complement of $V$. Then $W$ is characteristic in $V$ and so it is normal in $G$. Therefore we have Claim (d) by Claim (c).

Claim (e). We have $G_{p} \neq H_{1}$.

Assume that $G_{p}=H_{1}$. Let $Z$ be a subgroup of order $p$ in $Z\left(G_{p}\right) \cap D$. Then, since $D \leq O^{\sigma_{1}}(G)=O^{p}(G), Z$ is normal in $G$ by Lemma 2.5. Hence we have 
$D=Z<G_{p}$ and so $D<C_{G}(D)$. Then $V=C_{G}(D) \cap M \neq 1$ is a normal subgroup of $G$ and $V \leq H_{1}=G_{p}$ by Claim (d). Let $L$ be a minimal normal subgroup of $G$ contained in $V$. Then $G_{p}=D \times L$ is a normal elementary abelian subgroup of $G$ by Claim (c). Hence every maximal subgroup of $G_{p}$ is normal in $G$ by Lemmas 2.6 (3) and 2.5. It follows that every subgroup of $G_{p}$ is normal in $G$. Hence $|D|=|L|=p$. Let $D=\langle a\rangle, L=\langle b\rangle$ and $N=\langle a b\rangle$. Then $N \not \subset D$, so in view of the $G$-isomorphisms

$$
D N / D \simeq N \simeq N L / L=G_{p} / L=D L / L \simeq D
$$

we get that $G / C_{G}(D)=G / C_{G}(N)$ is a $p$-group since $G / D$ is $\sigma$-nilpotent by Lemma 2.1. But then Claim (d) implies that $G$ is a $p$-group. This contradiction shows that we have Claim (e).

Final contradiction for Claim 5. In view of [15, Theorem A], $G$ has a $\sigma_{1}$-complement $E$ such that $W=E G_{p}=G_{p} E$. Let $V=W^{\mathfrak{N}_{\sigma}}$. In view of Claims 2 and 4 and Lemma 2.6 (5), the hypothesis holds for $W$. Moreover, Claim (e) implies that $W \neq G$. Hence the conclusion of the theorem holds on $W$ by the choice of $G$, which implies that $V$ is a Hall subgroup of $W$. Moreover, Claim 4 implies that $V \leq D$, so for a Sylow $p$-subgroup $V_{p}$ of $V$ we have $\left|V_{p}\right| \leq|P|<\left|G_{p}\right|$. Hence $V$ is a $p^{\prime}$-group and so $V \leq C_{G}(D) \leq H_{1} \cap W$ by Claim (d). Hence $V=1$. Therefore $W=E G_{p}=E \times G_{p}$ is $\sigma$-nilpotent and so $E \leq C_{G}(D) \leq H_{1}$. Hence $E=1$ and so $D=1$, a contradiction. Thus, $D$ is a Hall subgroup of $G$.

Claim 6. If $p$ is a prime such that $(p-1,|G|)=1$, then $p$ does not divide $|D|$. In particular, the smallest prime divisor of $|G|$ divides $|G: D|$.

Assume that this is false and let $P$ be the Sylow $p$-subgroup of $D$. Then, arguing similarly as in the proof of Claim 3 , one can show that some maximal subgroup $E$ of $P$ is normal in $G$. Hence $C_{G}(D / E)=G$ since $(p-1,|G|)=1$ by hypothesis. Since $D$ is a Hall subgroup of $G$ by Claim 5, it has a complement $M$ in $G$. Hence $G / E=(D / E) \times(M E / E)$, where $M E / E \simeq M \simeq G / D$ is $\sigma$-nilpotent. Therefore $G / E$ is $\sigma$-nilpotent. But then $D \leq E$, a contradiction. Hence $p$ does not divide $|D|$. In particular, the smallest prime divisor of $|G|$ divides $|G: D|$.

Claim 7. Every chief factor of $G$ below $D$ is cyclic.

Suppose that this is false. Assume that $\Phi(D) \neq 1$ and let $R \leq \Phi(D)$. Claim 1 implies that every chief factor of $G / R$ below $(G / R)^{\mathfrak{N}_{\sigma}}=D / R$ is cyclic, so every chief factor of $G$ below $D$ is cyclic by Lemma 2.8. Hence $\Phi(D)=1$, so every Sylow subgroup of $D$ is elementary. Moreover, there is a prime $p \in \pi(D)$ such that the Sylow $p$-subgroup $P$ of $D$ contains a minimal normal subgroup $N$ of $G$ 
such that $|N|>p$. Let $V$ be a maximal subgroup of $P$ such that $P=N V$. Then $N \cap V \neq 1$. Since $D$ is a Hall subgroup of $G, P \in \operatorname{Syl}_{p}(G)$. Therefore $V$ is $\tau_{\sigma}$-quasinormal in $G$, so $N \cap V$ is $\sigma$-quasinormal in $G$ by Lemma 2.6(2)-(3). Now, arguing similarly as in the proof of Claim 3, one can show that $N \cap V$ is normal in $G$. The minimality of $N$ implies that $N \cap V=1$, so $|N|=p$. This contradiction completes the proof of Claim 7.

Claims 3, 5, 6 and 7 show that the conclusion of the theorem holds for $G$, which contradicts the choice of $G$.

The theorem is proved.

Proof of Theorem A. It is enough to show that if $G$ is a $\sigma$-soluble $T \sigma T$-group and $G$ is not $\sigma$-nilpotent, then conditions (i)-(iv) hold on $G$. Suppose that this is false and let $G$ be a counterexample of minimal order. Let $\mathscr{H}=\left\{H_{1}, \ldots, H_{t}\right\}$. We can assume without loss of generality that $H_{i}$ is a $\sigma_{i}$-group for all $i=1, \ldots, t$.

Claim 1. Every subgroup $H$ of $G$ is $\tau_{\sigma}$-quasinormal in $G$.

It is enough to consider the case when $H$ is a maximal subgroup of $G$. But since $G$ is $\sigma$-soluble by hypothesis, $|G: H|$ is a $\sigma_{i}$-number for some $i$ and so for a Hall $\sigma_{i}$-subgroup $H_{i}$ of $G$ we have $H H_{i}=G$. Hence $G=H H_{i}^{x}=H_{i}^{x} H$ for all $x \in G$. Thus we have Claim 1 .

Claim 2. We have $G=D \rtimes M$, where $D$ is nilpotent and condition (i) holds for $(D, M)$.

In view of the Schur-Zassenhaus theorem this directly follows from Theorem B and Claim 1.

Claim 3. Every subgroup of $D$ is normal in $G$. Hence $D$ is abelian and every element of $M$ induces a power automorphism in $D$.

In view of Lemma 2.10 this follows from Claims 1 and 2.

Claim 4. Condition (iv) holds on G.

First note that since $G$ is $\sigma$-soluble, it is a $\sigma$-full group of Sylow type by Lemma 2.4 and so every two Hall $\sigma_{k}$-subgroups of $G$ are conjugate for all $\sigma_{k} \in \sigma(G)$.

Since $G / D$ is $\sigma$-nilpotent, it follows that $D A$ and $D B$ are normal in $G$. Hence $A^{G}=A\left(A^{G} \cap D\right)$ and $B^{G}=B\left(B^{G} \cap D\right)$, so $A^{G} \cap B=1$ and $B^{G} \cap A=1$. Let $r \in \pi(D)$.

Claim (a). If $r \in \pi\left(A^{G}\right)$ and $C_{r}$ is a group of order $r$, then $C_{r} B A=A C_{r} B$ is a subgroup of $G$. If $r \in \pi\left(B^{G}\right)$, then $C_{r} A B=B C_{r} A$ is a subgroup of $G$. 
First note that $C_{r} \leq D$ since $r \in \pi(D)$ and $D$ is a Hall subgroup of $G$ by Claim 2. Therefore $C_{r}$ is normal in $G$ by Claim 3. Claim 1 implies that $C_{r} B$ is $\tau_{\sigma}$-quasinormal in $G$. On the other hand, we have $\sigma\left(C_{r} B\right) \cap \sigma(A)=\emptyset$ and also $\sigma\left(C_{r} B\right) \cap \sigma\left(A^{G}\right) \neq \emptyset$ since $r \in \pi\left(A^{G}\right)$, so $\left(C_{r} B\right) A=A\left(C_{r} B\right)$ is a subgroup of $G$. Similarly one can get the second assertion of Claim (a).

Claim (b). If $C_{r} \leq A^{G}$ and $C_{r} \not \leq B^{G}$ or $C_{r} \leq B^{G}$ and $C_{r} \leq A^{G}$, then $[A, B]=1$.

Assume, for example, that $C_{r} \leq A^{G}$ and $C_{r} \not \leq B^{G}$. Then

$$
\begin{aligned}
A^{G} \cap C_{r} B A & =C_{r}\left(A^{G} \cap B A\right) \\
& =C_{r}\left(A^{G} \cap B\right) A=C_{r} A,
\end{aligned}
$$

so $B \leq N_{G}\left(C_{r} A\right)$. On the other hand,

$$
\begin{aligned}
B^{G} \cap C_{r} B A & =B^{G} \cap A C_{r} B \\
& =\left(B^{G} \cap A C_{r}\right) B \\
& =\left(B^{G} \cap A\right)\left(B^{G} \cap C_{r}\right) B=B
\end{aligned}
$$

by Lemma 2.3, so $C_{r} A \leq N_{G}(B)$. Hence $[A, B] \leq\left[C_{r} A, B\right] \leq C_{r} A \cap B=1$.

Claim (c). If $C_{r} \leq A^{G} \cap B^{G}$ and $[A, B] \neq 1$, then $[A, B]=C_{r}$.

Claim (a) implies that $C_{r} B A$ and $C_{r} A B$ are subgroups of $G$, so

$$
C_{r} A B=C_{r} B A .
$$

Hence

$$
A^{G} \cap C_{r} A B=C_{r}\left(A^{G} \cap A B\right)=C_{r} A\left(A^{G} \cap B\right)=C_{r} A
$$

and $B^{G} \cap C_{r} A B=C_{r} B$, so $C_{r} B \leq N_{G}\left(C_{r} A\right)$ and $C_{r} A \leq N_{G}\left(C_{r} B\right)$. Hence we have $[A, B] \leq\left[C_{r} A, C_{r} B\right] \leq C_{r} A \cap C_{r} B=C_{r}$, so $[A, B]=C_{r}$ since $[A, B] \neq 1$.

Claim (d). If $C_{t} \leq[A, B]$, where $C_{t}$ is a group of prime order $t$, then we have $C_{t} \leq A^{G} \cap B^{G}, t \in \pi(D)$ and $[A, B]=C_{t}$. Moreover, if the Sylow $t$-subgroup $P$ of $D$ is not cyclic, then $[A, P]=1=[B, P]$.

Note that $C_{t} \leq A^{G} \cap B^{G}$ since $[A, B] \leq\left[A^{G}, B^{G}\right] \leq A^{G} \cap B^{G}$. But we have $A^{G} \cap B^{G}=A\left(A^{G} \cap D\right) \cap B\left(B^{G} \cap D\right)$. Hence $t \in \pi(D)$, so from Claim (c) we get $[A, B]=C_{t}$. Suppose that $P$ is not cyclic. Then, by [8, Chapter I, Section 2.20], $P$ possesses a subgroup $C_{1} \neq C_{t}$ of order $t$ since the smallest prime divisor of $|G|$ divides $|M|$ by Claim 2. Claim (c) implies that $C_{1} \not \leq A^{G}$ or $C_{1} \not \leq B^{G}$ 
since $[A, B]=C_{t}$. Hence we have $C_{1} \not \leq A^{G}$ and $C_{1} \not \leq B^{G}$ by Claim (b). Then $\left[C_{1}, A\right]=\left[C_{1}, B\right]=1$. But every element $a \in A$ induces a power automorphism $\alpha$ in $P$, so in the case when $\alpha$ is non-trivial, it is fixed-point-free by [13, Section 13.4.3 (ii)]. Therefore every element of $A$ acts trivially on $P$, so $[A, P]=1$. Similarly we get $[B, P]=1$.

Finally, suppose that $|[A, B]|=r$ is a prime and, also, $A$ is a $p$-group and $B$ is a $q$-group for some primes $p$ and $q$. Then $[A, B] \leq D$ and $E=[A, B] A B$ is a subgroup of $G$ by Claims (a) and (d). Let $V$ be a Hall $\{p, q\}$-subgroup of $E$. Then $V \simeq D V / D$ is nilpotent, so $E$ is supersoluble. Assume that $p>r$. Then $A$ or $B$ is normal in $E$ and so either $[A, B] \leq A$ or $[A, B] \leq B$, contrary to the fact that $1<[A, B] \leq D$. Hence $r>p$. Similarly we get that $r>q$. Therefore we have (iv).

Thus conditions (i)-(iv) hold on $G$, contrary to our assumption on $G$. The theorem is proved.

Acknowledgments. The authors are grateful to the helpful suggestions of the referee.

\section{Bibliography}

[1] A. Ballester-Bolinches, R. Esteban-Romero and M. Asaad, Products of Finite Groups, Walter de Gruyter, Berlin, 2010.

[2] A. Ballester-Bolinches and L. M. Ezquerro, Classes of Finite Groups, Springer, New York, 2006.

[3] X. Chen, W. Guo and A. N. Skiba, Some conditions under which a finite group belongs to a Baer-local formation, Comm. Algebra 42 (2014), 4188-4203.

[4] K. Doerk and T. Hawkes, Finite Soluble Groups, Walter de Gruyter, Berlin, 1992.

[5] W. Guo, Structure Theory for Canonical Classes of Finite Groups, Springer, Berlin, 2015.

[6] W. Guo and A. N. Skiba, Finite groups with permutable complete Wielandt sets of subgroups, J. Group Theory 18 (2015), 191-200.

[7] W. Guo and A. N. Skiba, On $\sigma$-semipermutable subgroups of finite groups, preprint (2016), http://arxiv.org/abs/1609.08815v1.

[8] B. Huppert, Endliche Gruppen I, Springer, Berlin, 1967.

[9] O. H. Kegel, Produkte nilpotenter Gruppen, Arch. Math. 12 (1961), 90-93.

[10] V. N. Knyagina and V.S. Monakhov, On $\pi^{\prime}$-properties of finite groups having a Hall $\pi$-subgroup, Siberian Math. J. 522 (2011), 398-309. 
[11] V. O. Lukyanenko and A. N. Skiba, On weakly $\tau$-quasinormal subgroups of finite groups, Acta Math. Hungar. 125 (2009), no. 3, 237-248.

[12] V. O. Lukyanenko and A. N. Skiba, Finite groups in which $\tau$-quasinormality is a transitive relation, Rend. Semin. Mat. Univ. Padova 124 (2010), 1-15.

[13] D. J. S. Robinson, A Course in the Theory of Groups, Springer, New York, 1982.

[14] L. A. Shemetkov, Formations of Finite Groups, Nauka, Moscow, 1978.

[15] A. N. Skiba, A generalization of a Hall theorem, J. Algebra Appl. 15 (2015), no. 4, 21-36.

[16] A. N. Skiba, On $\sigma$-subnormal and $\sigma$-permutable subgroups of finite groups, J. Algebra 436 (2015), 1-16.

[17] A. N. Skiba, On some results in the theory of finite partially soluble groups, Commun. Math. Stat. 4 (2016), no. 3, 281-309.

[18] S. Srinivasan, Two sufficient conditions for supersolubility of finite groups, Israel J. Math. 3 (1980), no. 35, 210-214.

Received December 22, 2016.

\section{Author information}

James C. Beidleman, Department of Mathematics, University of Kentucky, Lexington, KY 40506-0027, USA.

E-mail: james . beidleman@uky . edu

Alexander N. Skiba, Department of Mathematics and Technologies of Programming, Francisk Skorina Gomel State University, Gomel 246019, Belarus.

E-mail: alexander.skiba49@gmail.com 